\title{
The effect of NET-1 on the proliferation, migration and endocytosis of the SMMC-7721 HCC cell line
}

\author{
GUI-LAN WANG $^{1 *}$, LI CHEN $^{1 *}$, YING-ZE WEI $^{2}$, JIA-MING ZHOU ${ }^{1}$, \\ YUAN-YUAN WU ${ }^{1}$, YI-XIN ZHANG ${ }^{2}$, JING QIN $^{1}$ and YUAN-YUAN ZHU ${ }^{3}$ \\ ${ }^{1}$ Department of Pathological Anatomy, Nantong University; ${ }^{2}$ Department of Pathology, \\ Nantong Tumor Hospital; ${ }^{3}$ Biomics (Nantong) Co., Ltd., Nantong, P.R. China
}

Received December 16, 2011; Accepted February 1, 2012

DOI: $10.3892 /$ or.2012.1698

\begin{abstract}
To explore the effect of NET-1 on the proliferation, migration and endocytosis in the hepatocellular carcinoma (HCC) cell line SMMC-7721, we constructed the pU6H1NET-1 siRNA (NET-1 siRNA) and pcDNA3.1/myc-NET-1 (myc-NET-1) vectors and transfected them into SMMC-7721 cells. The expression levels of NET-1 mRNA and protein were detected using real-time quantitative RT-qPCR and western blotting. The proliferation rates of SMMC-7721 cells were determined by CCK- 8 assays, flow cytometry (FCM) and immunohistochemistry staining. The migration in two or three dimensional space of SMMC-7721 cells were determined by wound-healing assay and in vitro invasion assay. The extent of endocytosis in SMMC-7721 cells was estimated by observing the amount of transferrin (Tfn) absorbed with capture ELISA assays, and Tfn endocytosis was observed under confocal immunofluorescence microscopy. The results show that: i) after transfecting NET-1 siRNA, the expression of NET-1 mRNA and protein in SMMC-7721 cells decreased significantly, the growth of cells was suppressed, which induced cell cycle arrest, the proliferation rates were dramatically reduced and the expression of Ki67 declined, and migration and endocytosis in cells were inhibited, compared with untreated cells (every $\mathrm{P}<0.01$ ); ii) Following transfection with myc-NET-1, the expression of NET-1 mRNA and protein in SMMC-7721 cells increased, and both the proliferation of cells and the cell cycle were promoted $(\mathrm{P}<0.01$, respectively). However, the abilities of cell migration and endocytosis were not affected compared with untreated cells. These data suggest that: i) the NET-1 gene may play an important role in proliferation, migration and endocytosis of cells; ii) siRNA technology may efficiently
\end{abstract}

Correspondence to: Li Chen, Department of Pathological Anatomy, Nantong University, 19, Qixiu Road, Nantong 226001, P.R. China E-mail: bl1@ntu.edu.cn

*Contributed equally

Key words: NET-1, hepatocellular carcinoma SMMC-7721 cells, proliferation, migration, endocytosis suppress the expression and function of NET-1 in HCC, suggesting that NET-1 may be a therapeutic target for HCC.

\section{Introduction}

Liver cancer is one of the most frequent causes of cancer death. An estimated 748,300 new liver cancer and 695,900 cancer deaths worldwide occurred in 2008. Half of these cases and deaths were estimated to occur in China (1). Among primary liver cancers, hepatocellular carcinoma (HCC) represents the major histological subtype, accounting for $70-85 \%$ of the total liver cancer burden worldwide (2). Despite aggressive surgical treatment, most patients still inevitably die from recurrence and metastasis of tumor. Qidong county of Nantong city in Jiangsu province in China has a high incidence of liver cancer. The average annual morbidity and mortality of liver cancer $(60-70 / 100,000)$ far exceeds the national average (3). So far, the molecular mechanism of the generation and development especially recurrence and metastasis of HCC is unknown. Therefore it is important to explore and study the relationship between the function of new genes and the generation and development of HCC, which may reveal its molecular mechanism, especially mechanism of the invasion and metastasis of $\mathrm{HCC}$, in order to seek new drug targets and improve the treatment level of HCC.

NET-1 gene, together with NET-2 to NET-7 genes, called NET-x, belong to the tetraspan superfamily (TM4SF) and was founded by Serru from EST database in the year 2000 (4). NET-1 is located at 1p34.1 chromosome. The full-length NET-1mRNA (Genebank accession no.: AF065388) has 1297 bp and an open reading frame of 241 amino acids. NET-1 is also termed C4.8, P50S, Tspan-1 (5).

Structurally TM4SF is a specific family member of cell membrane glycoprotein and widely expressed in many tissues and cells. The main characteristic is the existence of four hydrophobic regions delimiting extracellular transmembrane regions of cysteine residues, mediating extracellular signal transduction and playing an important role in cell development, growth and movement. Among these molecules, CD9, CD37, CD53, CD63, CD81/TAPA-1, CD82/KAI 1 and CD151/PETA-3 have been proved to be correlated with cancers (5-7).

In previous studies, we found that NET-1, a new gene of TM4SF is highly expressed in HCC $(8,9)$, but the role of NET-1 
in HCC is unclear and whether NET-1 affects the proliferation and invasion of cancer cells is also uncertain. Therefore, in this study, we explore the role of NET-1 in the HCC cells by observing the proliferation, migration and endocytosis of cancer cells.

\section{Materials and methods}

Plasmid construction. The siRNA sequences were designed on the basis of the published NET-1 sequence from GenBank (accession no.: AF065388) using Qiagen siRNA software (Qiagen, Shanghai, China). The 21nt NET-1 gene siRNA sequence GTTGGCAGTGGGCATCTGGG was cloned into pU6H1-GFP vector (5.5 kb, Qiagen) to construct siRNA expression plasmid targeting NET-1 (pU6H1-GFP-NET-1 siRNA, NET-1 siRNA). The full-length human NET-1 cDNA sense sequence was 5'-ATGCAGTGCTTCAGCTTCATTAA-3', and the antisense sequence was 5'-TTATTGTAGATTG CAGTACAGATAC-3', (726 bp), which was amplified and cloned into pUC57 cloning Vector (2.71 kb, Qiagen), then subcloned into pcDNA3.1/myc expression vector $(5.5 \mathrm{~kb}$, Qiagen) to construct the expression plasmid of NET-1 (pcDNA3.1/myc-NET-1, myc-NET-1). Simultaneously, the corresponding vector pU6H1-GFP or pcDNA3.1/myc was used as a negative control (mock) and untransfected cells were used as a blank control. The selected sequences were submitted to BLAST analysis (http://www.ncbi.nlm.nih.gov/blast/) to ensure that the selected gene was specifically targeted. These recombinant plasmids were constructed and sequenced by Biomics Biotechnologies Co., Ltd., Nantong, JS, China). pU6H1-GFP vector containing green fluorescent protein (GFP) was used to assay the function of the transfected Plasmid. siRNAs plasmid targeting Lamin gene (provided by Biomics Biotechnologies Co. Ltd.) was used as a positive control to confirm the feasibility of delivery, transfection, and reaction condition environments of siRNAs.

Cell culture and transfection. The human HCC cell line, SMMC-7721, was purchased from the Liver Cancer Institute, Zhongshan Hospital, Fudan University, Shanghai in China, and cultured at $37^{\circ} \mathrm{C}$ in a humidified incubator $\left(5 \% \mathrm{CO}_{2}\right)$ in RPMI1640 medium (Invitrogen, Carlsbad, CA, USA) supplemented with $10 \%$ heat-inactivated fetal calf serum (FCS), $100 \mathrm{U} / \mathrm{ml}$ penicillin, and $100 \mathrm{~g} / \mathrm{ml}$ streptomycin.

SMMC 7721 cells $\left(1.5 \times 10^{5}\right.$ cells per well) were plated in 6 -well plates (Nunc, Rochester, NY, USA). When the cells reached $70 \%$ confluence, they were transfected with the plasmids using Lipofectamine 2000 ${ }^{\mathrm{TM}}$ (Invitrogen) in accordance with the manufacturer's protocol. At $48 \mathrm{~h}$ post-transfection, images were analyzed by direct fluorescence microscopy (Olympus, Beijing, China). The cells were divided into 5 groups: NET-1 siRNA and pU6H1-GFP as the negative control, NET-1-myc and pcDNA3.1/myc as the negative control, and untreated cells as a blank control.

Real-time quantitative polymerase chain reaction ( $R T$ - $q P C R)$. Total RNA was isolated from SMMC-7721 cells using TRIzol reagent (Invitrogen), and then submitted to a $25 \mu \mathrm{l}$ PCR reaction in the presence of $12.5 \mu \mathrm{l}$ of $2 \mathrm{X}$ Master Mix, $1 \mu \mathrm{l}$ of each primer mix $(10 \mu \mathrm{M} / \mathrm{ml}), 0.5 \mu \mathrm{l}$ of $50 \mathrm{X}$ SYBR-Green I and $4 \mu \mathrm{l}$
RNA according to One-step Quantace kit (Australia). The PCR mixtures were first subjected to $30 \mathrm{~min}$ at $42^{\circ} \mathrm{C}$ for reverse transcription and initially denatured for $10 \mathrm{~min}$ at $94^{\circ} \mathrm{C}$ and then to 45 cycles of amplification with the following cycling parameters: $20 \mathrm{sec}$ at $95^{\circ} \mathrm{C}, 30 \mathrm{sec}$ at $55^{\circ} \mathrm{C}$ and $30 \mathrm{sec}$ at $72^{\circ} \mathrm{C}$. The primer pairs for NET-1 gene were designed with Primer Premier 5.0 software, glyceraldehyde-3-phosphate dehydrogenase (GAPDH) served as an internal control for PCR. The primer sequences for the genes and expected product sizes were as follows: 5'-GTGGCT TCACCAACTATACG-3' (forward), 5'-GACTGCATTAGTTCGGATGT-3' (reverse) for NET-1 (191 bp); 5'-TGATGACATCAAGAAGGTGGTGAAG-3' (forward), 5'-TCCTTGGAGGCCATGTGGGCCAT-3' (reverse) for GAPDH (240 bp).

Western blot assay. SMMC-7721 cells were lysed with radioimmunoprecipitation assay (RIPA) buffer (Sigma-Aldrich, St. Louis, MO, USA) $48 \mathrm{~h}$ after transfection and equal amounts of protein were separated by $10 \%$ SDS-PAGE and then transferred to a PVDF membrane. Nonspecific binding was blocked for $2 \mathrm{~h}$ with $5 \%$ nonfat milk in TBST (Tris-buffered saline containing $0.1 \%$ Tween-20). After incubation with the primary antibodies overnight at $4{ }^{\circ} \mathrm{C}$ (a rabbit anti-NET-1 polyclonal antibody at 1:200 dilution; the antibody was made by the authors in cooperation with Genemed Biotechnologies Inc, South San Francisco, CA, USA); a rabbit anti- $\beta$-actin antibody, at 1:2000 dilution (Sigma-Aldrich), membranes were washed 3 times in TBST for $5 \mathrm{~min}$ and subsequently incubated with a peroxidase-conjugated goat anti-rabbit secondary antibody [1:2500 dilution, (Sigma-Aldrich)] for $1 \mathrm{~h}$ at room temperature, and developed using a chemiluminescence system (Pierce, Rockford, IL, USA). The film was scanned and the density of the bands measured using Image Quant software (Molecular Dynamics, Sunnyvale, CA, USA), and then expressed as the percentage of the density of the $\beta$-actin band.

Immunofluorescence microscopy. SMMC-7721 cells were cultured on coverslips in 24-well plates, transfected, and $48 \mathrm{~h}$ later the coverslips were washed twice with phosphate-buffered saline (PBS), and fixed with $4 \%$ paraformaldehyde for $1 \mathrm{~h}$. The cells were permeabilized with $0.2 \%$ Triton X-100 for $5 \mathrm{~min}$, then blocked with $1 \% \mathrm{BSA}$ in PBS for $1 \mathrm{~h}$ at room temperature. Cells were incubated overnight at $4^{\circ} \mathrm{C}$ with the NET-1 primary antibody (1:200 dilution) and Mouse anti-human monoclonal antibody Ki67 (purchased from Zymed Co. USA, diluted 1:100), then washed extensively, followed by labeling with a secondary TRITC or FITC labeled antibody [(1:100 dilution (SigmaAldrich)] for $2 \mathrm{~h}$ at $37^{\circ} \mathrm{C}$. The nuclei were counterstained by Hoechst 33258 (5 $\mu \mathrm{g} / \mathrm{ml}$; Invitrogen) for $30 \mathrm{~min}$ and the stained cells observed using immunofluorescent microscopy.

Cell counting kit-8 (CCK-8) assay. For assaying SMMC-7721 cell growth, $100 \mu \mathrm{l}$ of a $2 \times 10^{4}$ cells $/ \mathrm{ml}$ suspension in DMEM complete medium was seeded per well of 96-well culture plates (Costar, Corning, NY, USA). At 1-5 days after transfection, $10 \mu \mathrm{l}$ of CCK-8 solution (Dojindo, Japan) was added to each well, followed by incubation at $37^{\circ} \mathrm{C}$ for $2 \mathrm{~h}$ away from light. The absorbance (OD values, reflecting the cell cycle) was detected at $450 \mathrm{~nm}$ using Microplate Reader (Bio-Rad 680). All samples were tested in triplicates, and the differences between 
A

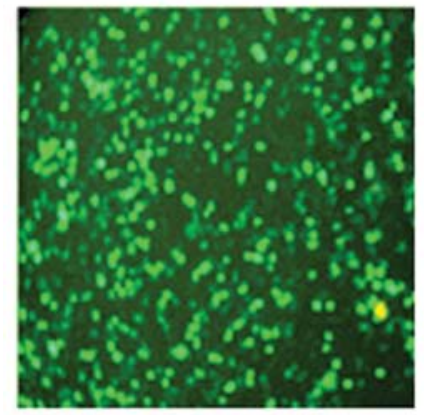

Ba

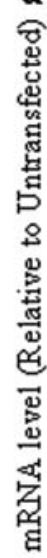

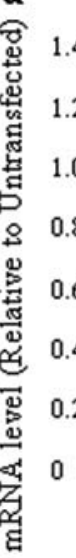

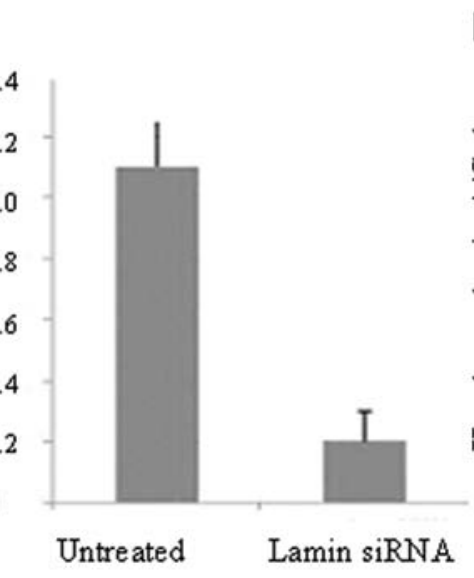

b

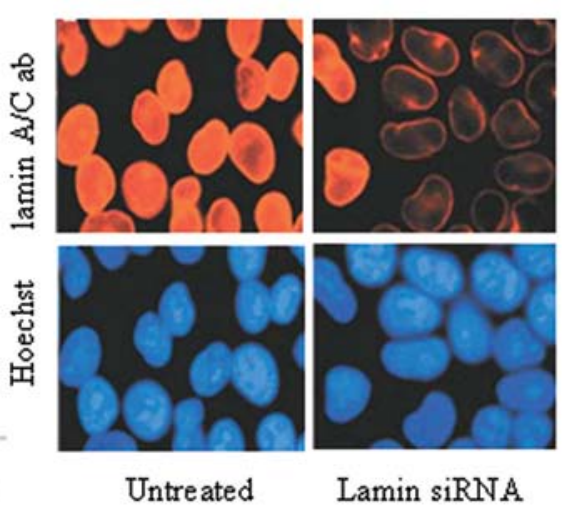

Figure 1. The result of transfection and silencing with siRNA in SMMC-7721 cells. (A) Fluorescence micrograph of fluorescein labeled GFP (green fluorescence in cytoplasm) in SMMC-7721 cells at $48 \mathrm{~h}$ after transfection with siRNA (unfixed cells, original magnification x200), the arrows point to the fluorescein positive cells. The transfection efficiency was $\sim 80 \%$. (B) The expression level of Lamin gene silencing was $>80 \%$, (a) histogram of expression levels, (b) fluorescence micrograph of TRITC labeled Lamin (red fluorescence).

the controls and the test groups were analyzed. The OD value was the mean of eight wells.

Cell cycle analysis by flow cytometry. After $48 \mathrm{~h}$ in culture, transfected SMMC-7721 cells $\left(1 \times 10^{6}\right)$ were washed twice with ice-cold PBS, harvested by trypsinization, and then fixed in $70 \%$ cold ethanol at $4^{\circ} \mathrm{C}$ overnight. The cell pellets were resuspended in a staining solution of $0.1 \%$ Triton-X, DNase-free Rnase and propidiumiodide (Sigma-Aldrich) for $30 \mathrm{~min}$ at room temperature in the dark. Flow-activated cell sorter analysis was carried out using a Calibur flow cytometer (BD Biosciences, San Jose, CA, USA) and CellQuest software. Finally, proliferation ratio (PI) of each group of cells was calculated by the formula: $\mathrm{PI}=(\mathrm{S}+\mathrm{G} 2 / \mathrm{M}) /(\mathrm{G} 0 / \mathrm{G} 1+\mathrm{S}+\mathrm{G} 2 / \mathrm{M})$.

Wound-healing assay. SMMC-7721 cells were cultured in 96-well plates to monolayer, and tips were used to scratch across the bottom of each well. Cells were washed twice with serum-free DMEM medium gently to remove dead cells. Then, $100 \mu \mathrm{l}$ of complete DMEM medium with $10 \%$ FBS was added to each well for continuous culture. Images were taken at 0 , 24,48 and $72 \mathrm{~h}$ in the same position and magnification using Image-ProPlus software (version 4.5.1, USA). The following formula was used to calculate relative migration distance: relative migration distance $=(\mathrm{A}-\mathrm{B}) / \mathrm{A} \times 100$. A represents the initial scratching distance, and $\mathrm{B}$ is the distance in each experiment.

In vitro invasion assay. The invasive potential of SMMC-7721 cells was determined by an invasion assay using polycarbonate membranes (8.0- $\mu$ mpore size) in the upper half of 24-well Transwell culture chambers coated with Matrigel (Costar). After transfection for $24 \mathrm{~h}, \mathrm{SMMC}-7721$ cells $\left(1 \times 10^{5}\right)$ were suspended with $100 \mu \mathrm{l}$ serum-free RPMI-1640 medium and placed in the upper chamber. The lower compartment of the chamber was filled with $500 \mu 1 \mathrm{RPMI}-1640$ medium containing serum. After $24-\mathrm{h}$ incubation in $5 \% \mathrm{CO}_{2}$ at $37^{\circ} \mathrm{C}$, nonmigratory cells on top of the filters were gently removed with cotton cotton swabs. The invading cells on the underside of the filter were fixed for $10 \mathrm{~min}$ in $10 \%$ formaldehyde, stained with $1 \%$ crystal violet for $5 \mathrm{~min}$, and washed with PBS. The number of cells was quantified by counting the cells in at least 5 random fields per filter (magnification, $\mathrm{x} 200$ ).

Capture ELISA assays. After transfected, SMMC-7721 cells were cultured for $30 \mathrm{~min}$, the $5 \mathrm{mg} / \mathrm{l}$ biotinylated transferrin (bio-Tfn, Pierce) was added, and then cells were placed on ice to continue to culture for $30 \mathrm{~min}$. Cells were lysed with blocking buffer (1\% BSA, $1 \%$ TritonX-100, $10 \mathrm{mM}$ Tris-HCl, $\mathrm{pH} 7.5,0.1 \%$ SDS, $1 \mathrm{mM}$ EDTA, $50 \mathrm{mM} \mathrm{NaCl}$ ), and next, cell lysates were added to 96-well plate packaged with anti-Tfn antibody (Pierce; diluted 1:50). The cells were then incubated at $4^{\circ} \mathrm{C}$ for $12 \mathrm{~h}$. Finally each well was tested for OD450 values for intracellular bio-Tfn levels by adding HRP compound.

Transferrin endocytosis assay. SMMC-7721 cells were seeded into 24-well plates and treated as described. Twenty-four hours later, the cells were changed to serum-free DMEM medium and incubated for $1 \mathrm{~h}$, before the medium was changed to $200 \mu \mathrm{l}$ treatment medium supplemented by $4 \mu \mathrm{l}$ bio-Tfn and $10 \%$ FBS. The cells were incubated for $1 \mathrm{~h}$ at $37^{\circ} \mathrm{C}$, washed once by PBS, fixed with $4 \%$ paraformaldehyde for $30 \mathrm{~min}$. Cells were incubated overnight at $4^{\circ} \mathrm{C}$ with the anti-Tfn antibody (1:100 dilution), then washed extensively, followed by a secondary TRITC labeled antibody [(1:100 dilution (SigmaAldrich)] for $2 \mathrm{~h}$ at $37^{\circ} \mathrm{C}$. The nuclei were counterstained by Hoechst 33258 (5 $\mu \mathrm{g} / \mathrm{ml}$, Invitrogen) for $30 \mathrm{~min}$. Confocal immunofluorescence microscopy was performed on a BioRad MRC1024 system, and the images were captured at x630 magnification with the aid of Laser Shaqi software for visualizing transferrin.

Statistic analysis. All experiments were performed a minimum of 3 separate times with at least 3 replicates per experiment (except CCK-8 experiment which was performed in octads). Data are presented as means \pm SD, and analyzed by SPSS 13.0 (SPSS Inc., Chicago, IL, USA). A Mann-Whitney U test was 

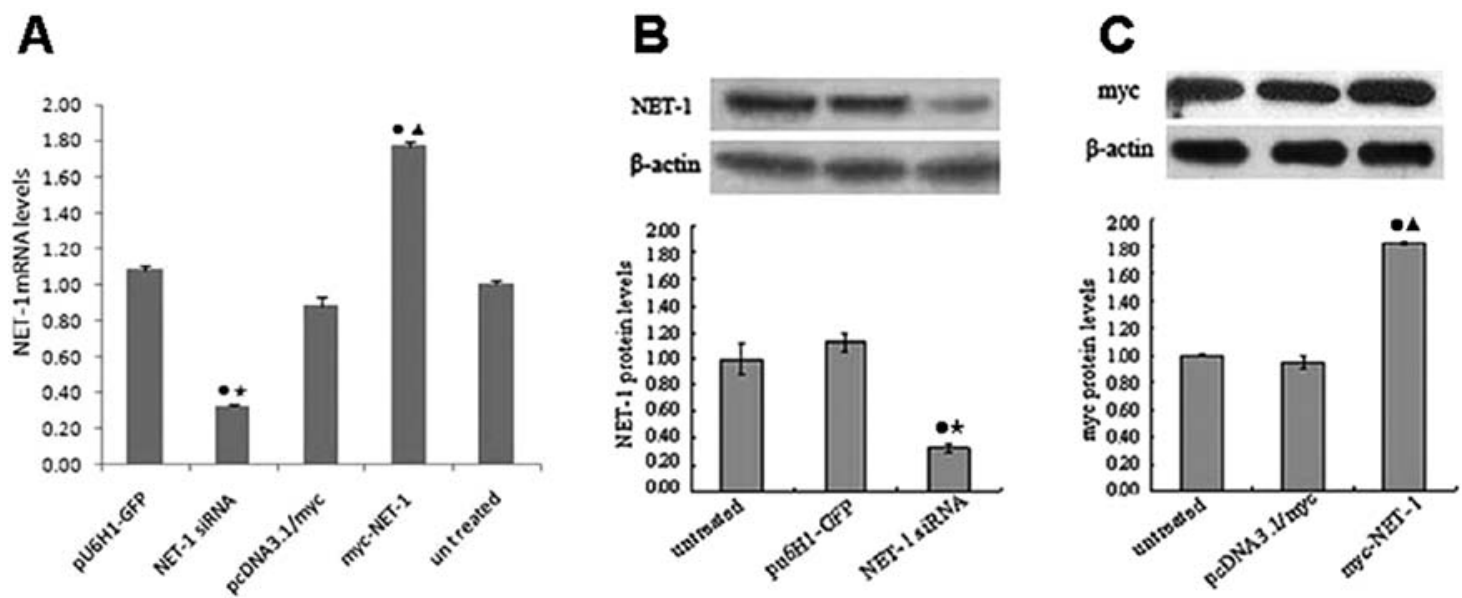

Figure 2. The expression of NET-1 mRNA and protein in SMMC-7721 cells. (A) Expression of NET-1 mRNA levels in SMMC-7721 cells with either NET-1 siRNA or myc-NET-1 transfected by RT-qPCR. NET-1 mRNA levels in cell transfection with NET-1 siRNA resulted in 68\% reduction compared with untreated cell group and 70\% reduction compared with the pU6H1-GFP (mock) $(\mathrm{T}=-10.213$ and 17.545, $\mathrm{P}<0.01$, respectively). Transfection of cells with myc-NET-1 resulted in 77\% improvement compared with the untreated cell group and 98\% improvement compared with pcDNA3.1/myc (mock) (T= -11.027 and $-22.250, \mathrm{P}<0.01$, respectively). (B) The expression levels of NET-1 protein in SMMC-7721 cells with NET-1 siRNA transfected by western blotting. NET-1 protein expression reduced significantly by $74.5 \%$ in transfected NET-1 siRNA group compared with pU6H1-GFP group and $66.1 \%$ compared with the untreated group ( $\mathrm{T}=-34.341$ and $-14.414, \mathrm{P}<0.001$, respectively). $\beta$-actin was included as a control in the experiments. (C) The expression levels of myc protein in SMMC-7721 cells with myc-NET-1 transfected by western blotting. Significant increase of $72.6 \%$ in myc-NET-1 compared with the untreated cell group and $74.2 \%$ compared with pcDNA3.1/myc (mock) $(\mathrm{T}=62.873$ and 38.662, $\mathrm{P}<0.001$, respectively). $\beta$-actin was included as a control in the experiments. The untreated cell group was set as 1. $\bullet$ NET-1 siRNA group or myc-NET-1 group were compared with the untreated group; $\star$, NET-1 siRNA group compared with the pU6H1-GFP (mock); $\mathbf{\Lambda}$, myc-NET-1 group compared with the pcDNA3.1/myc (mock).

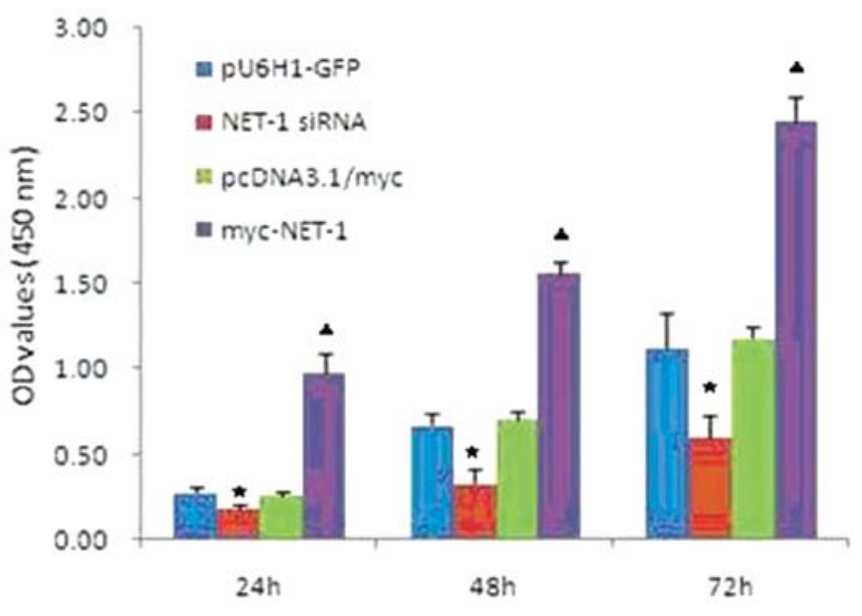

Figure 3. The effect of NET-1 to the proliferation of SMMC-7721 by CCK-8. $\star$, NET-1 siRNA group was compared with the pU6H1-GFP at 24,48 and $72 \mathrm{~h}(\mathrm{t}=13.190,29.290$ and 10.498 , respectively) each $\mathrm{P}<0.01 ; \mathbf{\Lambda}$, myc-NET-1 group compared the pcDNA3.1/myc group $(t=39.602,26.969$ and 20.937 , respectively) each $\mathrm{P}<0.01$.

used to measure statistical significance between experimental groups. $\mathrm{P}<0.05$ was considered statistically significant.

\section{Results}

DNA sequencing and the efficiency of cell transfection. Sequencing confirmed the constructed NET-1 siRNA plasmid contained the U6 promoter and $\mathrm{H} 1$ promoter, and $21 \mathrm{nt}$ of siRNA NET-1-specific sequence was introduced between two promoters via PCR. Eukaryotic expression myc-NET-1 plasmid was $100 \%$ homologous with the human NET-1 gene. DNA sequencing proved the construction, and design of
NET-1 siRNA and myc-NET-1 expression plasmids was kept consistent.

Forty-eight hours after transfection pU6H1-GFP vector, GFP was observed in cells through fluorescence microscopy, and the efficiency of the cell transfection was $\sim 80 \%$ (Fig. 1A). After $72 \mathrm{~h}$ of transfection, the GFP expression gradually decreased. After transfection of siRNAs targeted Lamin, the expression levels of Lamin gene silenced significantly (Fig. 1B) to confirm the feasible delivery, transfection and reaction condition environments of siRNAs.

The expression of NET-1 gene. RT-qPCR and western blot analyses were performed to determine whether transfection with either NET-1 siRNA or myc-NET-1 would result in either reduction or increasing of NET-1 mRNA and protein in cells (Fig. 2). Compared to the untreated group, the level of NET-1 mRNA was significantly downregulated to $68 \%$ in NET-1 siRNA transfected cells, however, upregulated to $77 \%$ in Myc-NET-1 transfected cells (Fig. 2A). Gene silencing at protein level was $66.1 \%$ decreased in NET-1 siRNA-treated cells, and $72.6 \%$ increased in myc-NET-1-treated cells (Fig. 2B). There was no significant difference in NET-1 mRNA and protein levels between the untreated cell group and the mock groups (each $\mathrm{P}>0.05$ ).

Effect of NET-1 on SMMC-7721 cell proliferation. CCK-8 assay result showed that cell proliferation in NET-1 siRNA group was lower than that in pU6H1-GFP group in different times, but in myc-NET-1 was significantly higher than that in pcDNA3.1/myc group (Fig. 3). FCM analyzed and demonstrated the cell cycle distribution of SMMC-7721 cells after 48-h transfection of NET-1 siRNA and myc-NET-1 (Fig. 4). To examine the relationship between NET-1 and cell proliferation, the expression of Ki67 and NET-1 was further detected 

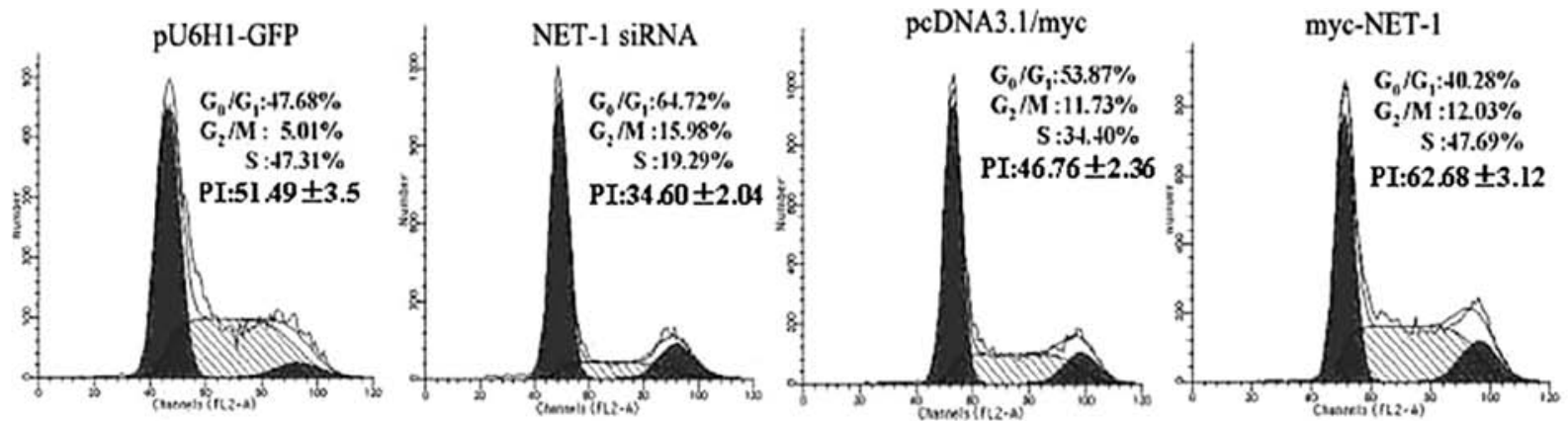

Figure 4. The effect of NET-1 on the cell cycle of SMMC-7721 cells by FCM. After $48 \mathrm{~h}$ of transfection, the proportion of G0/G1 and G2/M in NET-1 siRNA group increased by 17.04 and 10.97\% compared to that in pU6H1-GFP cells group, respectively; the proportion of S phase decreased in the former (19.29\%) compared with the latter $(47.31 \%)(\mathrm{P}<0.05)$. Cells in myc-NET-1 group entered into the cell cycle faster than that in pcDNA3.1/myc group, the proportion of G0/G1 phase decreased significantly in the former $(40.28 \%)$ compared with the latter $(53.87 \%)(\mathrm{P}<0.05)$. Comparison of PI results, there were significant differences between pU6H1-GFP group and the NET-1 siRNA group $(\mathrm{t}=17.290, \mathrm{P}=0.003)$ and between pcDNA3.1/myc group and myc-NET-1 group $(\mathrm{t}=-13.264, \mathrm{P}=0.006)$.
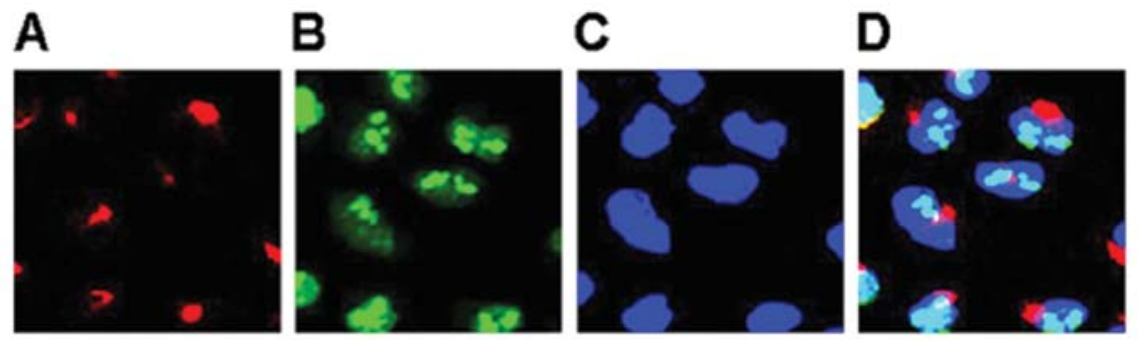

untreated
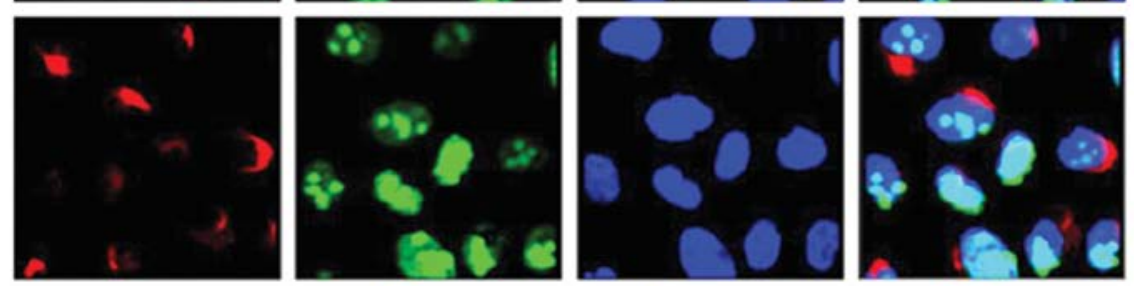

pU6H1-GFP
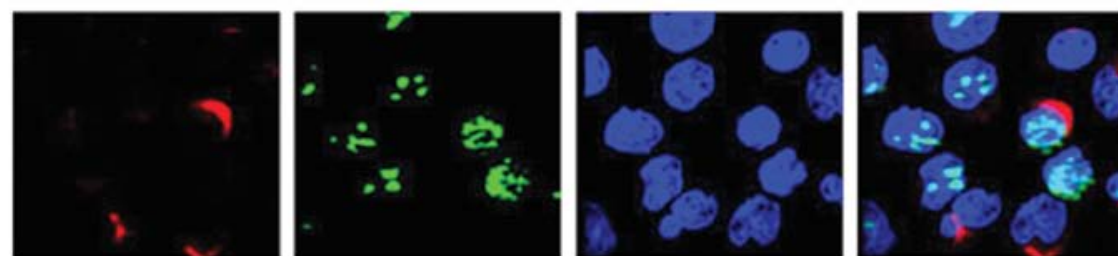

NET-1 1 SiRNA
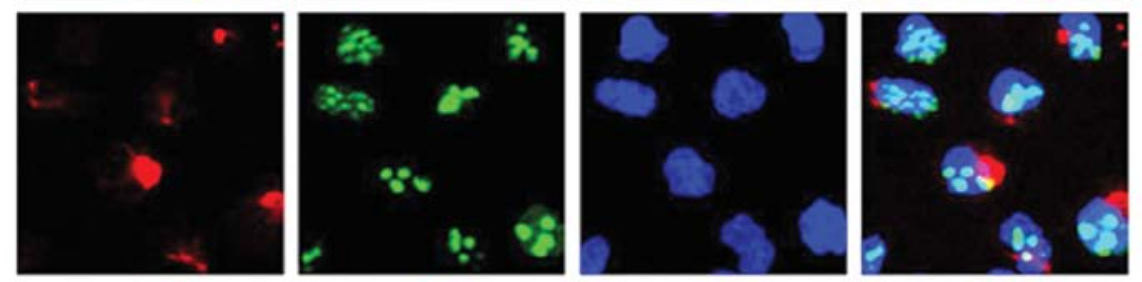

pcDNA3.1/ myc
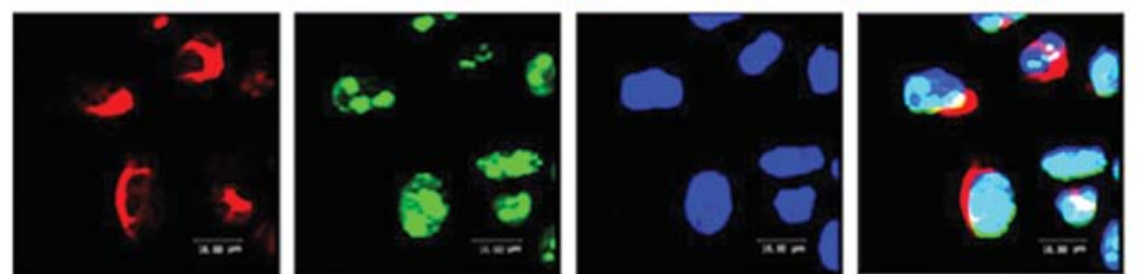

myc-NET-1

Figure 5. NET-1 and Ki 67 expression in SMMC-7721 cells. NET-1 is expressed evenly in a dot-like manner, as a small polar concentration in the cytoplasm near the nucleus, stained by TRITC in red (A), Ki67 located in nuclei stained by FITC in green (B) and the nuclei stained by Hochest in blue (C), respectively. Merged image (D).

by immunofluorescence assay and observed under a confocal microscope. The results indicated that NET-1 protein distributed evenly in a dot-like manner, as a small polar concentration in the cytoplasm near the nucleus of SMMC-7721 cells. Ki67 located in the nuclei of SMCC-7721 cells. A significantly reduced NET-1 signal was observed in NET-1 siRNA 


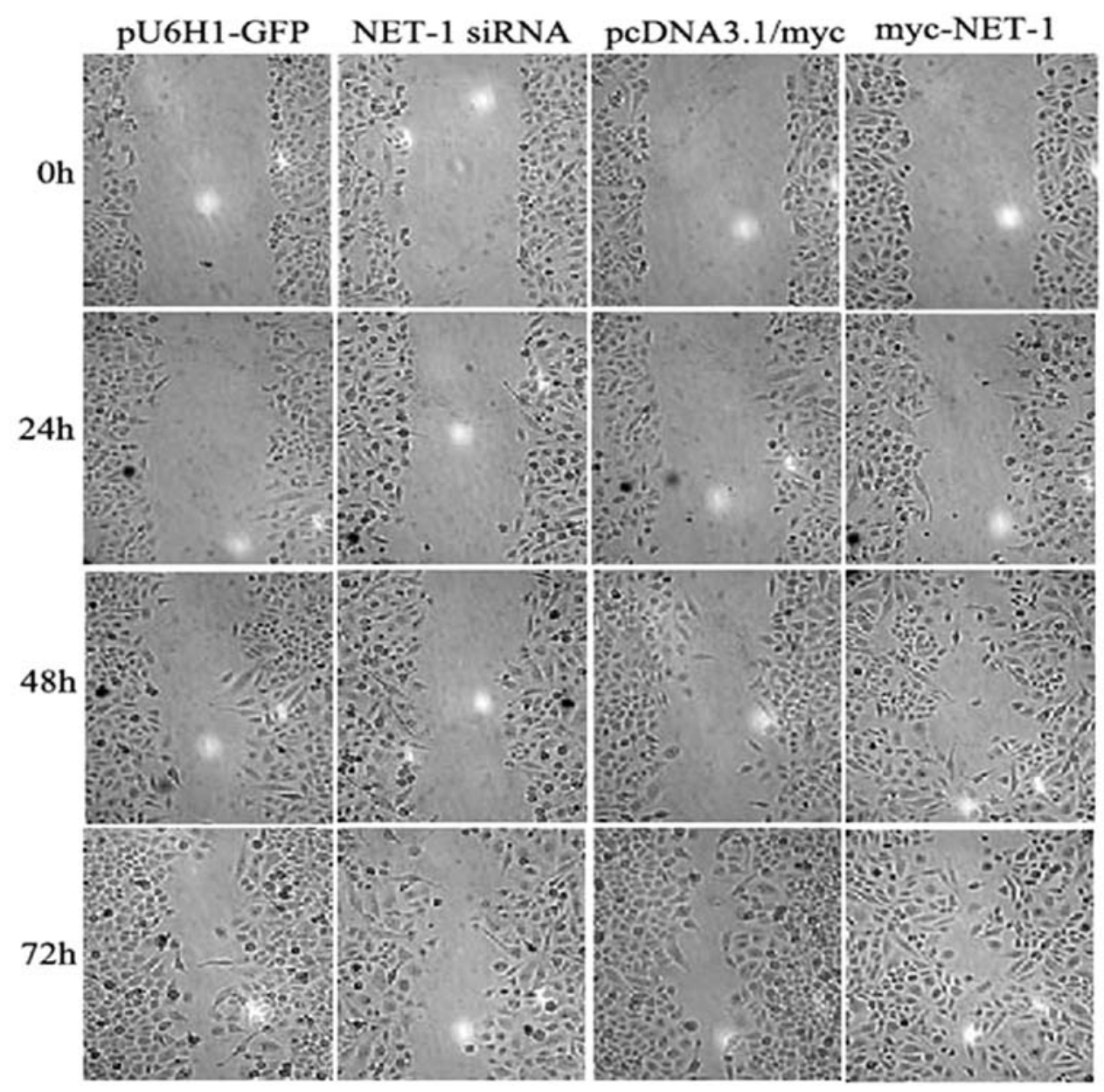

Figure 6. Cell migration was tested after transfection by Wound-healing assay (x100).

Table I. The migration amount of SMMC-7721 cells at different time after transfection.

\begin{tabular}{lcccccc}
\hline \multirow{2}{*}{$\begin{array}{l}\text { Observation } \\
\text { time }\end{array}$} & \multicolumn{5}{c}{ Migration cells, means \pm SD } & t value \\
\cline { 2 - 6 } & pU6H1-GFP & NET-siRNA & t value & pcDNA3.1/myc & myc-NET-1 & -1.1750 \\
\hline $24 \mathrm{~h}$ & $18 \pm 1.23$ & $16 \pm 0.60$ & 2.5312 & $19 \pm 1.53$ & $21 \pm 2.52$ & $-11.495^{\mathrm{a}}$ \\
$48 \mathrm{~h}$ & $25 \pm 1.53$ & $17 \pm 1.00$ & $13.856^{\mathrm{a}}$ & $33 \pm 2.52$ & $55 \pm 2.65$ & -1.867 \\
$72 \mathrm{~h}$ & $74 \pm 4.36$ & $39 \pm 3.21$ & $20.207^{\mathrm{a}}$ & $89 \pm 4.58$ & $95 \pm 3.61$ & \\
\hline
\end{tabular}

${ }^{\mathrm{a}} \mathrm{p}<0.01 ;$ (means $\pm \mathrm{SD}, \mathrm{N}=8$ ).

treated cells, and a significantly increased NET-1 signal in myc-NET-1, which was in accordance with Ki67 expression levels (Fig. 5). However, there was no significant alteration in the other groups. It was demonstrated that NET-1 gene related to cell proliferation.

Effect of NET-1 on SMMC-7721 cell migration. Woundhealing assay was performed to detect cells migration in two-dimensional space (Fig. 6). The amounts of cell migration were counted at different time points $(24,48$, and $72 \mathrm{~h})$ (Table I). The migration amount in NET-1 siRNA group was remarkably reduced compared to pU6H1-GFP group, in contrast, remarkably increased in myc-NET-1 group versus the pcDNA3.1/myc group. There were statistically significant differences after $48 \mathrm{~h}$ of transfection in each comparison.

Invasion assay was performed to detect migration in threedimensional space (Fig. 7). The cell amounts invading through the chamber membrane in different groups were observed after transfection of $48 \mathrm{~h}$. The average number of migrating cells in the NET-1 siRNA, pU6H1-GFP, myc-NET-1 and pcDNA3.1/myc cells was $21.42 \pm 5.74,45.60 \pm 6.14,64.80 \pm 6.14$ and 38.20 \pm 7.22 , respectively. This showed that the ability of cells transfected with NET-1 siRNA to invade was remarkably reduced as compared with pU6H1-GFP group ( $\mathrm{T}=-20.785, \mathrm{P}=0.002)$. In contrast, myc-NET-1 was remarkably increased as compared with $\mathrm{pcDNA} 3.1 / \mathrm{myc}(\mathrm{T}=5.027, \mathrm{P}=0.037)$. 
A
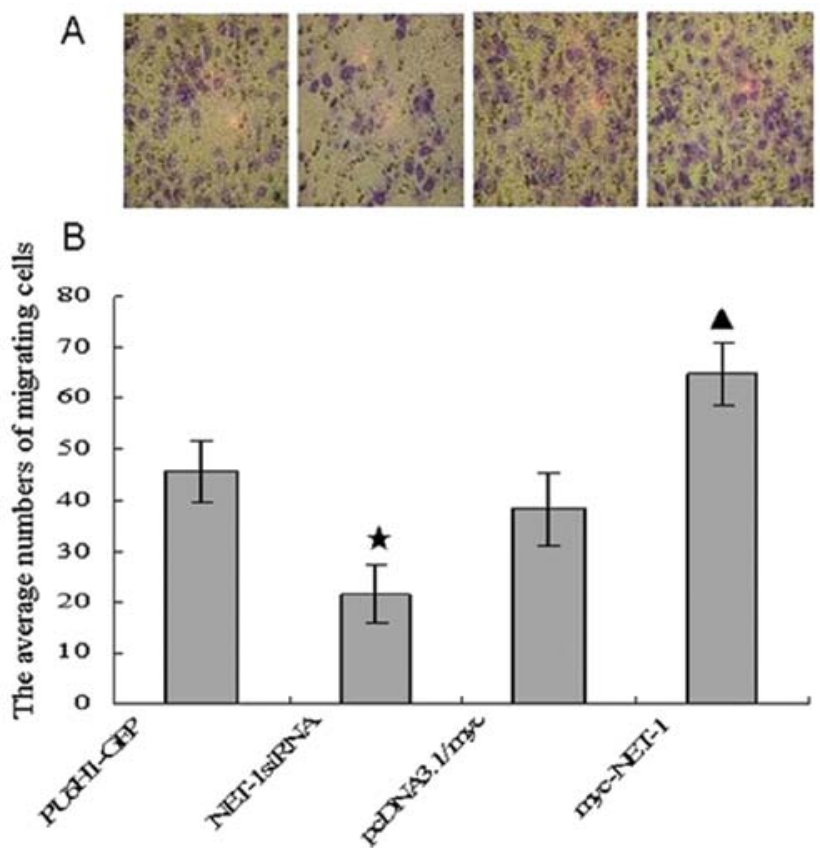

Figure 7. The invaded cells in the transwell assay after $48 \mathrm{~h}$ transfection (x200). The invading cells on the underside of the filter were stained with crystal violet (A) and quantified (B). $\star$, NET-1 siRNA group compared with the PU6H1-GFP group, $\mathrm{P}<0.01 ; \boldsymbol{\Lambda}$, myc-NET-1 group compared with the pcDNA3.1/myc, $\mathrm{P}<0.05$.

Effect of NET-1 on SMMC-7721 cell endocytosis. Transferrin endocytosis experiment demonstrated that endocytosis in NET-1 siRNA group was reduced as compared with untreated cell group and pU6H1-GFP $(\mathrm{t}=-26.739$ and 12.588 , respectively, $\mathrm{P}<0.01)$. Cell endocytosis morphology, by visualizing transferrin was observed in confocal immunofluorescence microscopy (Fig. 8B), which showed transferrin distributed mainly in the cytoplasm and significantly reduced in NET-1 siRNA treated cells as compared with other groups. However, there were no significant differences among other groups.

\section{Discussion}

The therapeutic approach for HCC can vary widely depending on the extent of disease including potentially curative surgical resection of small localized tumors, liver transplantation, nonsurgical invasive therapies, such as radiofrequency ablation (RFA) and transarterial chemoembolization (TACE) and newer biologic therapies for more advanced disease (10). However, each approach has its limits. SiRNA-targeted silencing of the genes associated with tumor cell proliferation or metastasis, as one method of gene therapy, shows great potency on HCC treatment. Highly-selective, sequence-specific and highly-effective characteristics make RNAi technology suitable to treat HCC with fewer adverse effects.

NET-1 gene, a member of TM4SF, may play an important role in multiplicative cellular functions, such as proliferation, migration and endocytosis of cells, and be regulated by intracellular molecular mechanisms. The mechanism of tetraspanins affecting tumor cell behavior has been studied in recent years. Tetraspanins form complexes by interacting with other tetraspanins and with a variety of transmembrane and cytosolic proteins that are required for their functioning $(11,12)$. The mechanism of NET-1 expression, regulation and the action of its products has not been clarified, which needs further study at the gene and protein levels.

Previous studies suggested NET-1 was expressed highly in some human tumor cell lines and tissues. Xu et al (13) used

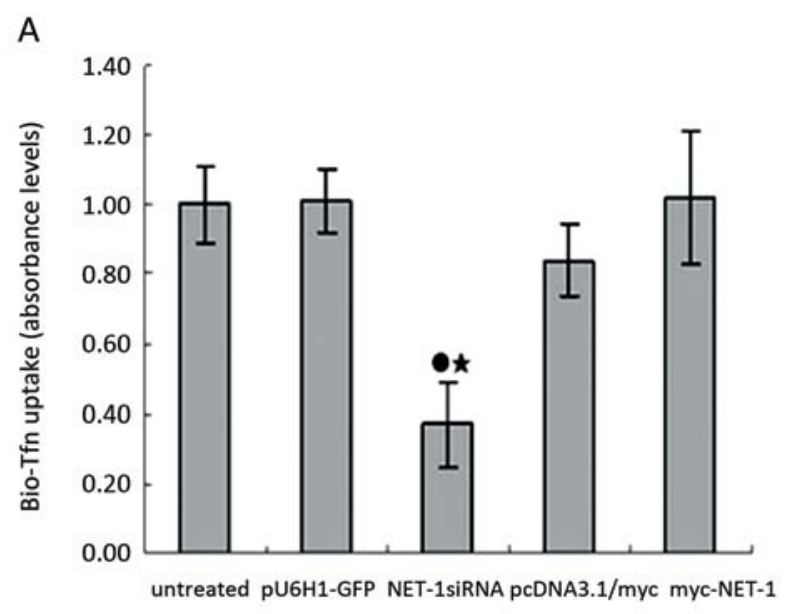

B
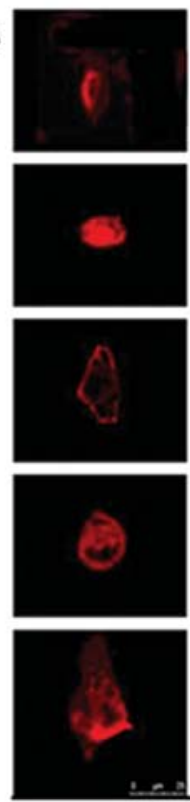
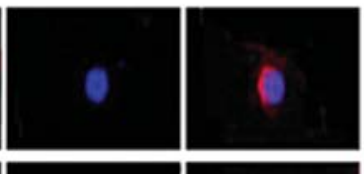

untreated
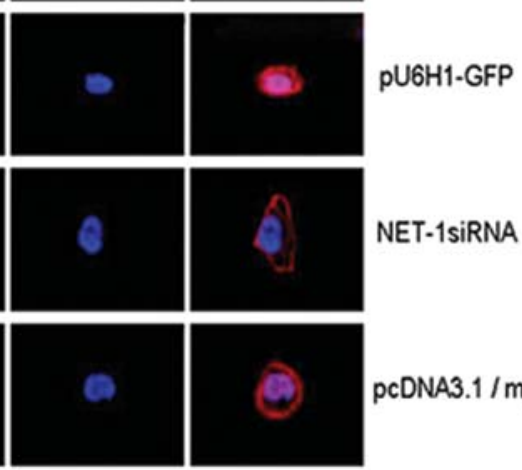

NET-1siRNA
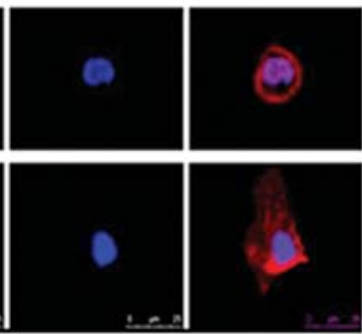

pcDNA3.1/myc

myc-NET-1

Figure 8. SMMC-7721 cell endocytosis in Tfn endocytosis experiment after $48 \mathrm{~h}$ transfection. (A) Capture-ELISA assays showed cell endocytosis in NET-1 siRNA group was reduced compared with the untreated cell group and pU6H1-GFP. Untreated cell group was set as 1 . $\bullet$, Either NET-1 siRNA group or myc-NET-1 group was compared with the untreated group $\mathrm{P}<0.01$; $\star$, NET-1 siRNA group compared with the pU6H1-GFP P $<0.01$. (B) Confocal immunofluorescence microscopy showed transferrin distributed in the cytoplasm was significantly reduced in NET-1 siRNA treated cells compared with other groups (each $\mathrm{P}<0.01$ ). 
cDNA library subtraction in conjunction with high throughput microarray screening to find NET-1 expression in prostate tumors and normal prostate tissue. Serru et al (4) reported NET-1 gene was expressed in many cancer cell lines through RT-PCR detection. In cervical cancer $(14)$, liver cancer $(8,9)$, colon cancer $(15,16)$, gastric carcinoma (17) and skin squamous cancer (16), NET-1 was unusually overexpressed. A series of studies confirmed a close relationship between NET-1 expression and the degree of tumor cell differentiation and malignancy. In this study, we investigated the effect of NET-1 on HCC cell lines (SMMC-7721) biological behavior, proliferation, migration and endocytosis.

The plasmids expressing NET-1 siRNA and myc-NET-1 were constructed to transfect HCC cell lines. The efficiency of transfection and silencing was estimated under fluorescence microscopy through observing GFP in cells transfected with pU6H1-GFP vector and Lamin expression in cells transfected with Lamin siRNAs. The results revealed that transfection efficiency and silencing efficiency was up to $80 \%$, suggesting a feasible delivery, transfection, and reaction condition environment of siRNAs for further experiments.

The expression of NET-1 mRNA and protein after transfection were detected by RT-qPCR and western blot analyses to confirm the efficiency of NET-1 siRNA and myc-NET-1. The results showed the expression of NET-1 mRNA and protein was reduced or increased in NET-1 siRNA group or myc-NET-1 group, respectively, as well as no significant difference in pU6H1-GFP or pcDNA3.1/myc group compared with untreated cell group. Furthermore, the effect of NET-1 on the biological behavior of HCC cell was investigated.

CCK-8 assay and FCM analyzed results showed that cell proliferation was inhibited and cell cycle was blocked at G0/G1 and $\mathrm{G} 2 / \mathrm{M}$, the proliferation rates were reduced significantly in NET-1 siRNA group. In contrast, in myc-NET-1 group, cell proliferation and cell cycle were significantly increased and promoted, which was in accordance with Ki67 expression levels in the cellular nuclei. It was demonstrated that NET-1 gene related to cell proliferation, the mechanism of which may be through alternating the progress of the cell cycle from G1 to $\mathrm{S}$ phase. We observed that NET-1 protein was located as a small polar concentration in the cytoplasm near the nucleus of cells, the area where the Golgi apparatus is frequently located. Thus, we suggest that NET-1 might regulate the proliferation of cancer cells by interacting with other transmembrane and cytosolic proteins when located on the membrane of secretion organelles, and carry out functions in the cytoplasm, like other tetraspanins.

A characteristic feature of any malignant tumor is the ability of tumor cells metastasis. In the present study, after transfecting NET-1 siRNA, the migration amounts of cells were remarkably reduced in either two- or three-dimensional space. Similar to our present findings (15-18), literature reported that siRNA-mediated downregulation of NET-1 expression resulted in decreased proliferation and invasion of colon cancer $(15,16)$ and gastric carcinoma (18) in vitro and in vivo.

Eukaryocytes carry out transmembrane transportation between large molecules and granules via endocytosis, performing also other functions (19), for example, nutrition uptake, growth factor regulation and signal transduction.
Endocytosis is a process through which extracellular materials are transported into cell through membrane deformation. This process is not a simple step-by-step process in which a series of proteins function according to the chronological order, but rather a complex process comprising many members precisely regulated. It has been reported that the abnormal expression of endocytosis might be involved in the mechanism of certain diseases, such as diabetes and neurological diseases and also closely related to the malignant transformation of cells. In this study, the results of endocytosis assay showed a significantly reduced transferrin in the cells transfected with NET-1 siRNA, which indicated that reducing NET-1 levels in cells, endocytosis was proved to be reduced, so it suggested that endocytosis of cells might to some extent relate with NET-1 gene, although the ability of cell endocytosis in myc-NET-1 was not significantly affected as compared with untreated cells. Whether tumor cells endocytose more nutrients than normal cells and whether tumor cells increase proliferation through endocytosing more growth factors is unclear. Can we inhibit tumor invasion and metastasis through inhibiting cell endocytosis or can we cure tumor by inducing specific drug endocytosis of tumor cells? The function of NET-1 gene can be further elucidated by solving the above problems.

The above results suggested that NET-1 gene may participate in the critical processes of proliferation, migration and endocytosis in the development and progress of $\mathrm{HCC}$, which helps to understand the associating molecular pathways involved in cell activities and malignant transformation and the mechanisms of HCC comprehensively. SiRNA technology targeting NET-1 gene may efficiently suppress the expression and function of NET-1 in HCC, suggesting NET-1 may be a therapeutic target for HCC. Further research in this area will be in the regulation mechanism governing cancer cell motility and the molecular pathway and to screen more effective siRNAs targeting NET-1.

\section{Acknowledgements}

This study is supported by a Project Funded by the Priority Academic Program Development of Jiangsu Higher Education Institutions, Foundation of the Ministry of Health, Jiangsu Province, China (No. H201052), and the Science Foundation of Nantong City, Jiangsu Province, China (No. K2009060). We thank Biomics Biotechnologies Co., Ltd. (Nantong, Jiangsu, China) for kindly providing viral plasmids.

\section{References}

1. Ferlay J, Shin HR, Bray F, Forman D, Mathers C and Parking DM: Estimates of worldwide burden of cancer in 2008: GLOBOCAN 2008. Int J Cancer 127: 2893-2917, 2008.

2. Perz JF, Armstrong GL, Farrington LA, Hutin YJ and Bell BP: The contributions of hepatitis $B$ virus and hepatitis $C$ virus infections to cirrhosis and primary liver cancer worldwide. J Hepatol 45: 529-538, 2006.

3. Lau WY, Leung TW, Lai BS, Leiw CT, Hb SK, Yu SC and Tang AM: Preoperative systemic chemoimmunotherapy and sequential resection for unresectable hepatocellular carcinoma. Ann Surg 233: 236-241, 2001.

4. Serru V, Dessen P, Boucheix C and Rubinstein E: Sequence and expression of seven new tetraspans. Biochim Biophys Acta 1478: $159-163,2000$. 
5. Iwai K, Ishii M, Ohshima S, Miyatake K and Saeki Y: Expression and function of transmembrane-4 superfamily (Tetraspanin) proteins in osteoclasts: reciprocal roles of Tspan-5 and NET-6 during osteoclastogenesis. Allergol Int 56: 457-463, 2007.

6. Maecker HT, Todd SC and Levy S: The tetraspanin superfamily: molecular facilitators. FASEB J 11: 428-442, 1997.

7. Claas C, Wahl J, Orlicky DJ, Karaduman H, Schnölzer M, Kempf T and Zöller M: The tetraspanin D6.1A and its molecular partners on rat carcinoma cells. Biochem J 389: 99-110, 2005.

8. Chen L, Yuan D, Wang GL, Wang Y, Wu YY and Zhu J: Clinicopathological significance of expression of Tspan-1, Jab1 and p27 in human hepatocellular carcinoma. J Korean Med Sci 25: $1438-1442,2010$

9. Chen L, Wang Z, Zhan X, Li DC, Zhu YY and Zhu J: Association of NET-1 gene expression with human hepatocellular carcinoma. Int J Surg Pathol 15: 346-353, 2007.

10. Wong $R$ and Frenette C: Updates in the management of hepatocellular carcinoma. Gastroenterol Hepatol 7: 16-24, 2011.

11. Hemler ME: Tetraspanin functions and associated microdomains. Nat Rev Mol Cell Biol 6: 801-811, 2005.

12. Levy S and Shoham T: Protein-protein interactions in the tetraspanin web. Physiology 20: 218-224, 2005.

13. Xu J, Stolk JA, Zhang X, Silva SJ, Houghton RL, Matsumura M, Vedvick TS, Leslie KB, Badaro R and Reed SG: Identification of differentially expressed genes in human prostate cancer using subtraction and microarray. Cancer Res 60: 1677-1682, 2000
14. Wollscheid V, Kühne-Heid R, Stein I, Jansen L, Köllner S, Schneider A and Dürst M: Identification of a new proliferationassociated protein INET-1/C4-8 characlerislie for a subset of high-grade cervical intraepithelial neoplasia and cervical carcinoma. Int J Cancer 99: 771-775, 2002.

15. Chen L, Zhu YY, Zhang XJ, Wang GL, Li XY, He S, Zhang JB and Zhu JW: TSPAN1 protein expression: A significant prognostic indicator for patients with colorectal adenocarcinoma. World J Gastroenterol 15: 2270-2276, 2009.

16. Chen L, Yuan D, Zhao R, Li H and Zhu J: Suppression of TSPAN1 by RNA interference inhibits proliferation and invasion of colon cancer cells in vitro. Tumori 96: 744-750, 2010.

17. Chen L, Li X, Wang GL, Wang Y, Zhu YY and Zhu J: Clinicopathological significance of overexpression of TSPAN1, Ki67 and CD34 in gastric carcinoma. Tumori 94: 531-538, 2008.

18. Chen L, Zhu Y, Li H, et al: Knockdown of TSPAN1 by RNA silencing and antisense technique inhibits proliferation and infiltration of human skin squamous carcinoma cells. Tumori 96: 289-295, 2010.

19. Chen L, Li H, Zhao R and Zhu JW: Study progress of cell endocytosis. Chinese-German J Clin Oncol 8: 360-365, 2009. 